\title{
Aquaporin-4-Deficient Mice Have Increased Extracellular Space without Tortuosity Change
}

\author{
Xiaoming Yao, ${ }^{1,2}$ Sabina Hrabětová, ${ }^{3,4}$ Charles Nicholson, ${ }^{3}$ and Geoffrey T. Manley ${ }^{1,2}$ \\ ${ }^{1}$ Department of Neurosurgery and ${ }^{2}$ Brain and Spinal Injury Center, University of California, San Francisco, San Francisco, California 94110, ${ }^{3}$ Department of \\ Physiology and Neuroscience, New York University School of Medicine, New York, New York 10016, and ${ }^{4}$ Department of Anatomy and Cell Biology, \\ Downstate Medical Center, State University of New York, Brooklyn, New York 11203
}

Aquaporin-4 (AQP4) is the major water channel expressed at fluid-tissue barriers throughout the brain and plays a crucial role in cerebral water balance. To assess whether these channels influence brain extracellular space (ECS) under resting physiological conditions, we used the established real-time iontophoresis method with tetramethylammonium (TMA ${ }^{+}$) to measure three diffusion parameters: ECS volume fraction $(\alpha)$, tortuosity $(\lambda)$, and TMA ${ }^{+}$loss $\left(k^{\prime}\right)$. In vivo measurements were performed in the somatosensory cortex of AQP4-deficient (AQP4 $\left.{ }^{-l-}\right)$ mice and wild-type controls with matched age. Mice lacking AQP4 showed a $28 \%$ increase in $\alpha(0.23 \pm$ 0.007 vs $0.18 \pm 0.003)$ with no differences in $\lambda(1.62 \pm 0.04 \mathrm{vs} 1.61 \pm 0.02)$ and $k^{\prime}\left(0.0045 \pm 0.0001 \mathrm{vs} 0.0031 \pm 0.0009 \mathrm{~s}^{-1}\right)$. Additional recordings in brain slices showed similarly elevated $\alpha$ in AQP4 ${ }^{-1-}$ mice, and no differences in $\lambda$ and $k^{\prime}$ between the two genotypes. This is the first direct comparison of ECS properties in adult mice lacking AQP4 water channels with wild-type animals and demonstrates a significant enlargement of the volume fraction but no difference in hindrance to $\mathrm{TMA}^{+}$diffusion, expressed as tortuosity. These findings provide direct evidence for involvement of AQP4 in modulation of the ECS volume fraction and provide a basis for future modeling of water and ion transport in the CNS.

Key words: aquaporin-4; diffusion; tetramethylammonium; volume fraction; seizure; water transport

\section{Introduction}

Aquaporin-4 (AQP4) is the major membrane water channel in the CNS. AQP4 is expressed in astrocyte foot processes in direct contact with capillary vessels in the brain (Frigeri et al., 1995; Nielsen et al., 1997). AQP4 is also expressed in the astrocytic processes that form the glia limitans, a structure adjacent to the CSF-filled subarachnoid space. Expression of AQP4 at these tissue-fluid interfaces indicates a role in maintaining brain water homeostasis. Phenotype studies of transgenic mice lacking AQP4 demonstrate its involvement in cerebral water balance and neural signal transduction (Manley et al., 2004). AQP4 ${ }^{-1-}$ mice have an increased seizure threshold (Binder et al., 2004a) and prolonged seizure duration with slowed $\mathrm{K}^{+}$kinetics in the brain extracellular space (ECS) (Binder et al., 2006). $\alpha$-Syntrophin-deficient mice, in which AQP4 is not properly targeted to the cell membrane, also show the deficit in $\mathrm{K}^{+}$clearance (Amiry-Moghaddam et al., 2003). These observations might be explained if ECS volume fraction $\alpha$ (defined as the ratio of ECS volume to that of whole tissue) were increased by AQP4 deletion (Manley et al., 2004), delaying the accumulation and clearance of neuroactive

\footnotetext{
Received Jan. 18, 2008; revised April 10, 2008; accepted April 14, 2008.

This work was supported by National Institutes of Health-National Institute of Neurological Disorders and Stroke Grants NS050173 and NS058931 (G.T.M.), NS047557 (S.H.), and NS28642 (C.N.).

Correspondence should be addressed to Dr. Geoffrey T. Manley, Department of Neurological Surgery, University of California, San Francisco, 1001 Potrero Avenue, Room 101, San Francisco, CA 94110. E-mail: manley@itsa.ucsf.edu.

DOI:10.1523/JNEUROSCI.0257-08.2008

Copyright $\odot 2008$ Society for Neuroscience $\quad$ 0270-6474/08/285460-05\$15.00/0
}

substances in the ECS. After birth, $\alpha$ is $\sim 0.4$ in rodent neocortex (Lehmenkühler et al., 1993) and shrinks to $\sim 0.2$ in the adult with a time course that matches glial proliferation and AQP4 expression (Wen et al., 1999). Consequently, it might be hypothesized that, in the absence of AQP4, this contraction of the ECS does not occur. Indeed, it has been claimed that the effective diffusion coefficient of dextran macromolecules increases in $\mathrm{AQP} 4^{-1-}$ mice (Binder et al., 2004b; Papadopoulos and Verkman, 2005; Zador et al., 2008), which these authors interpreted as implying an increase in $\alpha$. It was important, therefore, to directly test the hypothesis that $\alpha$ is enlarged in AQP4 ${ }^{-1-}$ mice and to obtain an absolute value for this and other diffusion parameters that might affect the role of AQP4 in brain water movement.

We used the real-time iontophoresis method with tetramethylammonium (RTI-TMA method) (Nicholson and Phillips, $1981)$ to measure $\alpha$ and tortuosity $\lambda$ [defined as $\left(D / D^{*}\right)^{1 / 2}$, where $D$ is the free diffusion coefficient of TMA ${ }^{+}$and $D^{\star}$ is the effective diffusion coefficient in brain] in $\mathrm{AQP} 4^{-/-}$and $\mathrm{AQP} 4^{+/+}$mice both in vivo and in vitro. We found a significant $28 \%$ increase in ECS volume fraction in AQP4-deficient mice with no change in tortuosity. These findings are discussed in the context of seizure threshold and provide a basis for future modeling of water and ion transport.

\section{Materials and Methods}

In vivo experiments took place at the University of California, San Francisco under a Committee on Animal Research-approved protocol. In vitro experiments were performed at New York University School of 
A

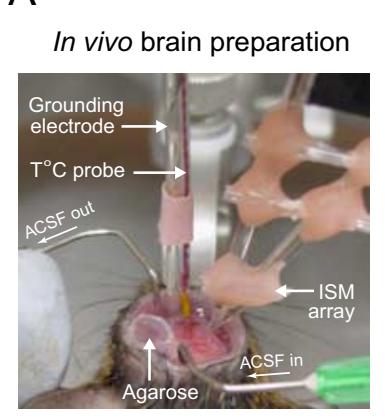

B

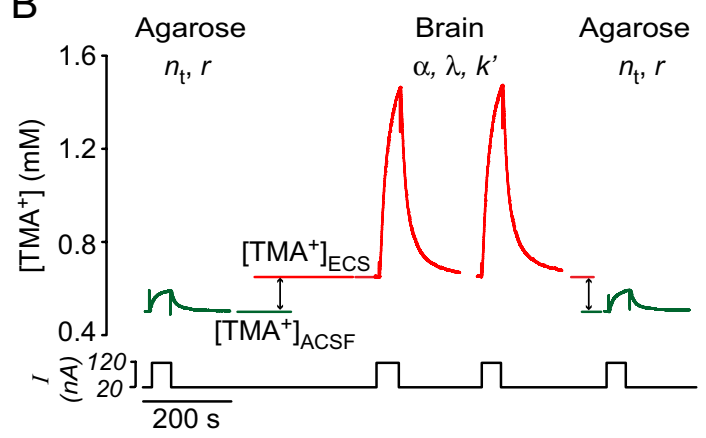

C
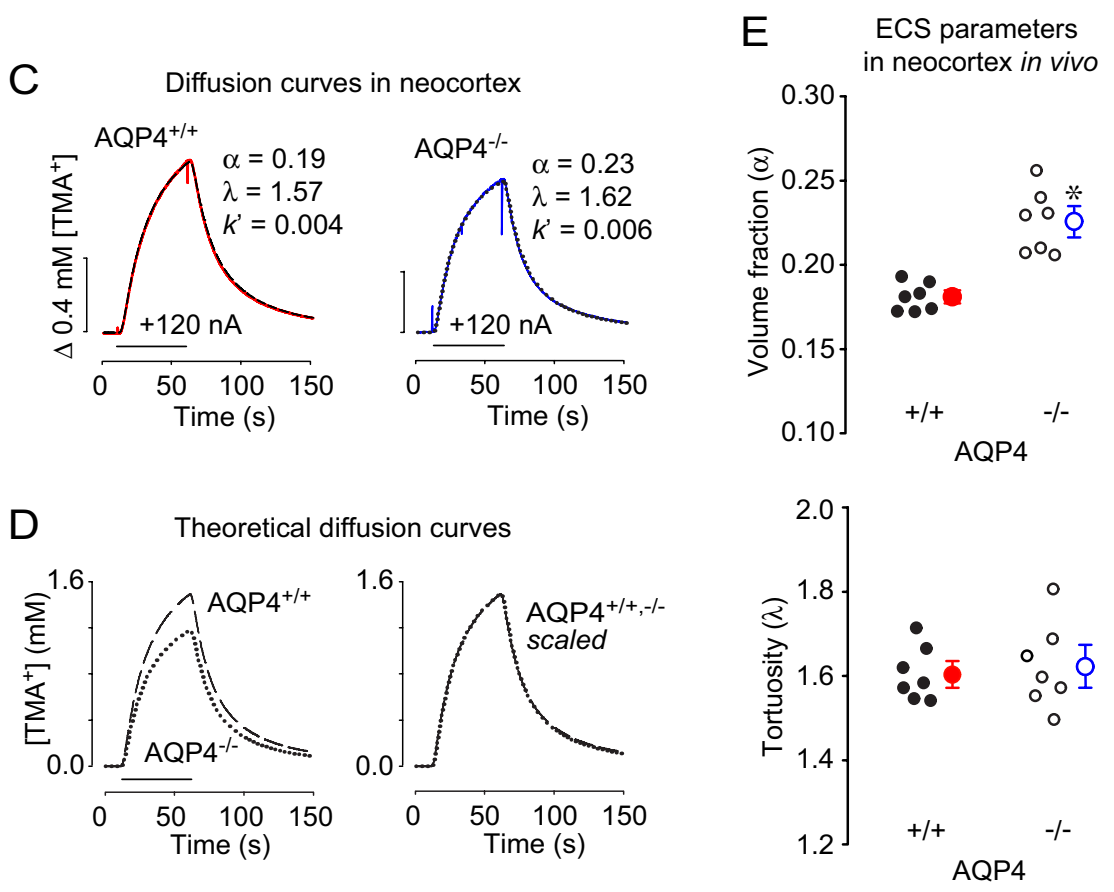

Figure 1. Diffusion of TMA ${ }^{+}$in the somatosensory neocortex of $\mathrm{AQP4} 4^{+/+}$and $\mathrm{AQP4} 4^{-/-}$mice in vivo. A, Photograph of recording arrangement for RTI-TMA diffusion measurements. The mouse head was immobilized in a stereotaxic frame. A micromanipulator positioned the microelectrodes that were glued with dental cement into an array with fixed intertip distance. The grounding electrode and the temperature probe were positioned nearby. See Materials and Methods for details. B, Sequence of diffusion curves recorded in dilute agarose gel and neocortex of $\mathrm{AQP4}{ }^{+/+}$mice. Several records were obtained in agarose before and after brain measurements. See Results for details. $C$, Examples of TMA ${ }^{+}$diffusion curves in AQP4 ${ }^{+/+}$and AQP4 ${ }^{-1-}$ mice. The measurements were done at $37^{\circ} \mathrm{C}$, where $D_{37}=1.31 \times 10^{-5} \mathrm{~cm}^{2} / \mathrm{s}$. TMA ${ }^{+}$pulse was applied for $50 \mathrm{~s}$ (horizontal bar), $r$ was 144 and $124 \mu \mathrm{m}$ for AQP4 ${ }^{+/+}$and AQP4 ${ }^{-/-}$, respectively, and $n_{\mathrm{t}}$ was 0.33 for both records. Recorded curves (red and blue) are superimposed with corresponding theoretical curves obtained from fitting procedure (dashed and dotted). $\boldsymbol{D}$, Diffusion curves obtained in $\mathrm{AQP4} 4^{+/+}$compared with those in $\mathrm{AQP} 4^{-1-}$ mice by generating theoretical curves based on the average $\alpha, \lambda$, and $k^{\prime}$ values (Table 1 and $\boldsymbol{E}$ ) with the following parameters: temperature, $37^{\circ} \mathrm{C}$; bias current, $+20 \mathrm{nA}$; main current, $+120 \mathrm{nA}$ for $50 \mathrm{~s} ; r=130 \mu \mathrm{m} ; n_{\mathrm{t}}=0.4$. The AQP4 ${ }^{-1-}$ theoretical curve had smaller amplitude than that for AQP4 ${ }^{+1+}$ animals (left), reflecting a larger $\alpha$, but both curves had similar shapes (right), indicating similar values of $\lambda$. $E$, Scatter plots of whole datasets. Each small circle represents the average from one animal. Large circles are mean \pm SEM values; ${ }^{*} p<0.001$.

Medicine in conformity with local Institutional Animal Care and Use Committee regulations.

Mice. AQP4 ${ }^{-1-}$ mice were generated in a $\mathrm{CD} 1$ genetic background (Ma et al., 1997). These mice lack detectable AQP4 protein and phenotypically had normal growth, development, survival, and neuromuscular function. Brains from wild-type and $\mathrm{AQP} 4^{-1-}$ mice showed no gross anatomical differences (Manley et al., 2000). Male AQP4 ${ }^{+/+}$and $\mathrm{AQP} 4^{-1-}$ mice were used with matched age and body weight (3-4 months, 30-35 g for the in vivo study and 4-4.5 months, 35-46 $\mathrm{g}$ for the in vitro). Twenty mice were used: seven AQP4 ${ }^{+/+}$and seven AQP4 $4^{-1-}$ for in vivo studies and three AQP4 ${ }^{+/+}$and three AQP $4^{-1-}$ mice for in vitro.

In vivo animal preparation. Mice were anesthetized using 2.5\% Avertin (2,2,2-tribromoethanol; $250 \mathrm{mg} / \mathrm{kg}, \quad$ i.p.; Sigma-Aldrich) and immobilized in a stereotaxic apparatus. An additional $85 \mathrm{mg} / \mathrm{kg}$ Avertin was given every hour to maintain anesthesia. After exposing the skull by midline skin incision, an atraumatic craniectomy was made over the somatosensory cortex $(1.5 \mathrm{~mm}$ lateral and $1.5 \mathrm{~mm}$ caudal to bregma) using a microdrill, and the dura was carefully removed. The skin flaps were held open with a cylindrical plastic dam (13 mm diameter and $4 \mathrm{~mm}$ height) (Fig. $1 A)$ and artificial CSF (ACSF; see below), at $37^{\circ} \mathrm{C}$, superfused over the brain at $2 \mathrm{ml} / \mathrm{min}$. A small plastic container $(4 \mathrm{~mm}$ diameter and 5 $\mathrm{mm}$ height) was glued to the inner wall of the dam to hold agarose gel for control measurements. The mice breathed room air spontaneously, and a heating pad maintained body temperature at $37 \pm 0.5^{\circ} \mathrm{C}$. Measurements were made at $1-3 \mathrm{~h}$ after surgery. The ACSF composition was as follows (in mM): $126 \mathrm{NaCl}, 3 \mathrm{KCl}$, $26 \mathrm{NaHCO}_{3}, 1.25 \mathrm{NaH}_{2} \mathrm{PO}_{4}, 10$ D-glucose, 1.3 $\mathrm{MgCl}_{2}$, and $1.5 \mathrm{CaCl}_{2}$, gassed with $95 \% \mathrm{O}_{2} / 5 \%$ $\mathrm{CO}_{2}$ to buffer $\mathrm{pH}$ at 7.4. For TMA ${ }^{+}$calibration, $0.5 \mathrm{~mm}$ TMA-chloride was added.

In vitro brain slices. Neocortical slices were prepared as described previously (Hrabětová et al., 2003). Briefly, animals were deeply anesthetized with sodium pentobarbital $(50 \mathrm{mg} / \mathrm{kg}$, i.p.) and decapitated, and the brain was removed from the skull and cooled with ice-cold ACSF. Coronal sections were cut at $400 \mu \mathrm{m}$ thickness with a vibrating blade microtome (VT 1000 S; Leica), submerged in ACSF, and incubated at room temperature for $1-6 \mathrm{~h}$ before measurements. The composition and gassing of the ACSF were the same as described above except that $\mathrm{NaCl}$ was $124 \mathrm{~mm}$ and $\mathrm{KCl}$ was $5 \mathrm{~mm}$.

For measurements, a brain slice was transferred to a submersion tissue chamber (model RC-27L; Warner Instruments) perfused at 2.0 $\mathrm{ml} / \mathrm{min}$ with ACSF warmed to $33 \pm 1^{\circ} \mathrm{C}$. The chamber was mounted on a Burleigh Gibraltar stage (EXFO Life Sciences) attached to an Olympus BX61WI microscope (Olympus America).

The RTI-TMA method. Microelectrodes for iontophoretic delivery and $\mathrm{TMA}^{+}$-ionselective microelectrodes (ISMs) were both pulled from double-barreled theta-glass (cata$\log$ \#64-0811; Warner Instruments) with a final outer diameter of $2-5 \mu \mathrm{m}$ and fabricated as described by Nicholson (1993). A tetraphenylborate-based ion exchanger (Corning 477317; currently available as IE190 from World Precision Instruments) was used in the ion-sensing barrel, which was backfilled with $150 \mathrm{~mm}$ TMA-chloride. The reference barrel contained $150 \mathrm{~mm} \mathrm{NaCl}$. To extract the $\mathrm{TMA}^{+}$signal, the voltage measured by the reference barrel was subtracted from the voltage measured by the ion-detecting barrel using a dual-channel microelectrode preamplifier (model IX2-700; Dagan). Each ISM was calibrated in solutions containing $0.5,1,2,4$, and 8 mM TMA-chloride in $150 \mathrm{~mm} \mathrm{NaCl}$, using the Nikolsky equation. The iontophoresis microelectrode contained $150 \mathrm{mM} \mathrm{TMA}^{+}$chloride.

Diffusion measurements in vivo. The shank of the iontophoretic microelectrode was bent and aligned parallel with the ISM, and both were glued together using dental cement with an intertip distance of 100-150 $\mu \mathrm{m}$ (Fig. $1 A$ ). Control TMA ${ }^{+}$diffusion curves were first recorded in agarose gel [0.3\% NuSieve GTG (FMC BioProducts) in $150 \mathrm{~mm} \mathrm{NaCl}$ and $0.5 \mathrm{~mm}$ 
TMA-chloride] to obtain the transport number $n_{\mathrm{t}}$ of the iontophoresis microelectrode and confirm the microelectrode spacing $r$, then measurements were made in brain to obtain the effective diffusion coefficient $D^{*}, \alpha, \lambda$, and the constant $k^{\prime}$ that accounted for nonspecific loss of $\mathrm{TMA}^{+}$from the ECS. A constant bias current of $+20 \mathrm{nA}$ was applied to the iontophoresis microelectrode from a high-impedance source (model ION-100; Dagan) to maintain a stable $n_{\mathrm{t}}(\mathrm{Ni}-$ cholson and Phillips, 1981). To generate a diffusion curve, an additional $100 \mathrm{nA}$ iontophoretic current pulse was applied every 3-5 min for $50 \mathrm{~s}$. The subtracted ion and reference signals were amplified and low-pass filtered $(4 \mathrm{~Hz})$ using a CyberAmp 380 (Axon-CNS; Molecular Devices). Diffusion curves were digitized and recorded with a personal computer, where they were converted to concentration using the ISM calibration and analyzed with the programs Wanda and Walter (Hrabětová and Nicholson, 2007). The electrode array was aligned along a transverse axis at a depth of $400 \mu \mathrm{m}$ in the somatosensory neocortex; because the neocortex is isotropic (Lehmenkühler et al., 1993), measurement along a single axis suffices to determine $\alpha$, $\lambda$, and $k^{\prime}$.

Diffusion measurement in brain slices. All recordings were made in somatosensory neocortical layers III-VI (Fig. 2A). The methods were similar to those used in vivo with the following changes. The iontophoretic microelectrode and the ISM were held in separate robotic micromanipulators (model MP 285; Sutter Instrument), with each microelectrode at an angle of $31^{\circ}$ from the horizontal plane (Fig. $2 \mathrm{~A}$ ) and the two electrode tips advanced into either agarose or cortical slice until they were $200 \mu \mathrm{m}$ deep and 130 $\mu \mathrm{m}$ apart. The iontophoresis current step varied from 30 to $100 \mathrm{nA}$.

Statistical analysis. Values are given as mean \pm SEM. Differences between groups were analyzed with a two-sample equal-variance $t$ test. Values of $p<0.05$ were considered significant.

\section{Results}

\section{ECS parameters in vivo}

Figure $1 \mathrm{~A}$ shows the experimental arrangement for in vivo diffusion measurements. A sequence of diffusion curves recorded in dilute agarose gel and in the somatosensory neocortex of AQP4 ${ }^{+/+}$mice is shown in Figure $1 B$. The first set of measurements in agarose gel provided the transport number, $n_{\mathrm{t}}$, of the iontophoretic microelectrode, and verified the intertip distance, $r$, of the micropipette array. Next, the array was positioned in the ACSF flowing above the brain to obtain a recording corresponding to $0.5 \mathrm{mM} \mathrm{TMA}^{+}$. Then the array was lowered into the brain, where, as shown in Figure $1 B,\left[\mathrm{TMA}^{+}\right]_{\mathrm{ECS}}>\left[\mathrm{TMA}^{+}\right]_{\mathrm{ACSF}}$ because the $20 \mathrm{nA}$ bias current applied to the iontophoresis microelectrode continuously released TMA ${ }^{+}$into the ECS. Several diffusion curves were taken in the neocortex, and finally, $\left[\mathrm{TMA}^{+}\right]_{\mathrm{ACSF}}$ was reverified, and records were taken again in agarose. Experiments using AQP4 ${ }^{-1-}$ mice were identical.

Figure $1 C$ shows representative diffusion curves superimposed with fitted theoretical curves for that record. To compare the overall diffusion curves from the two genotypes, we generated theoretical curves (Fig. 1D) based on averaged data (Table 1). The average diffusion curve in AQP4 ${ }^{-1-}$ mice had a smaller ammean \pm SEM values; ${ }^{*} p<0.001$.
B
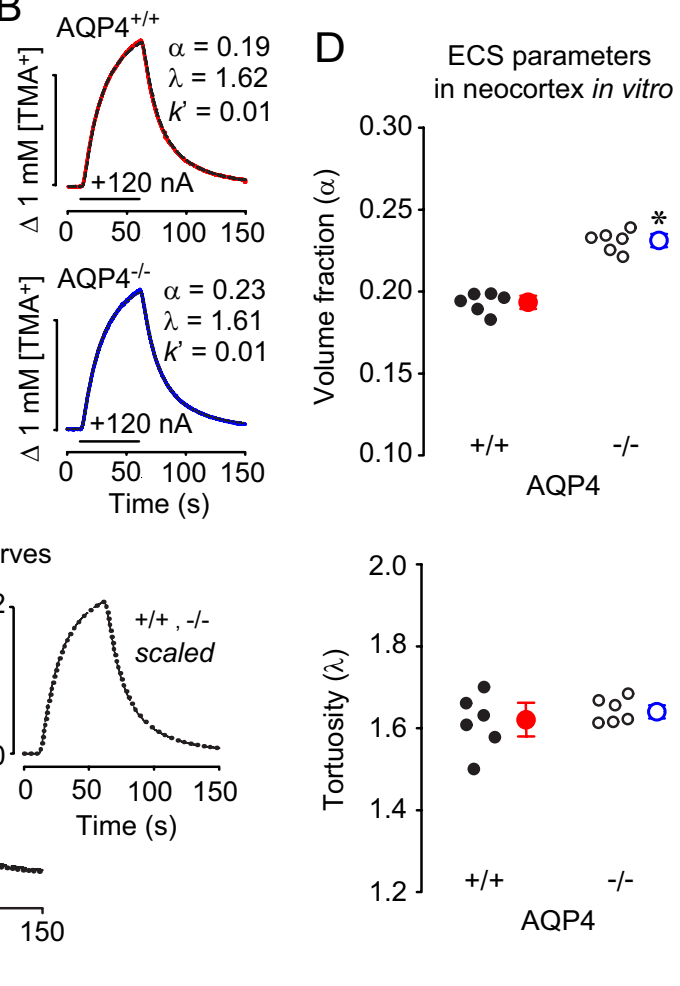

Figure 2. Diffusion of $\mathrm{TMA}^{+}$in the somatosensory neocortex (S1) of $\mathrm{AQP4} 4^{+/+}$and $\mathrm{AQP} 4^{-/-}$mice in vitro. $A$, A schematic of coronal brain slice with two independent microelectrodes positioned in S1 [Franklin and Paxinos (2008), their Figure 43 , modified with permission]. $\boldsymbol{B}$, Representative TMA ${ }^{+}$diffusion curves in $\mathrm{AQP4}{ }^{+/+}$(top) and $\mathrm{AQP4} 4^{-1-}$ (bottom) mice. The measurements were done at 34 and $32^{\circ} \mathrm{C}_{\text {in } \mathrm{AQP} 4}{ }^{+/+}$and $\mathrm{AQP4} 4^{-/-}$mice, respectively; $D_{34}=1.24 \times 10^{-5} \mathrm{~cm}^{2} / \mathrm{s}$ and $D_{32}=$ $1.19 \times 10^{-5} \mathrm{~cm}^{2} / \mathrm{s}$. TMA ${ }^{+}$pulse was applied for $50 \mathrm{~s}$ (horizontal bar), $r$ was $130 \mu \mathrm{m}$, and $n_{\mathrm{t}}$ was 0.39 and 0.48 for AQP4 ${ }^{+/+}$ mice, respectively. Actual $\mathrm{AQP4} 4^{+1+}$ record was taken with $60 \mathrm{nA}$ current but scaled to $120 \mathrm{nA}$ for consistency. In predure (dashed and dotted). C, As in Figure 1, to compare the diffusion properties in AQP4 ${ }^{+/+}$and AQP4 ${ }^{-1-}$ mice, the same parameters as in Figure but similar shape (inset). D, Scatter plots of entire datasets showing a significant increase in the volume fraction in AQP4 ${ }^{-1-}$ (top) but no change in the tortuosity (bottom). Each small circle represents the average from a single slice. Large circles are

plitude than that in AQP4 ${ }^{+/+}$animals, reflecting a larger volume fraction (Fig. 1D, left). In contrast, scaling the two curves to match amplitudes revealed similar shapes (Fig. $1 D$, right), demonstrating similar hindrance to $\mathrm{TMA}^{+}$diffusion (tortuosity) in $\mathrm{AQP} 4^{+/+}$and $\mathrm{AQP} 4^{-1-}$ animals.

Altogether, seven $\mathrm{AQP} 4^{+/+}$mice and seven $\mathrm{AQP} 4^{-/-}$mice were used. In each animal, up to seven diffusion curves were averaged to yield one set of $\alpha, \lambda$, and $k^{\prime}$ per animal, and summary values are shown in Figure $1 E$ and Table 1. These data indicate an enlarged ECS in the neocortex of AQP4 $4^{-1-}$ mice in vivo, whereas the tortuosity was similar in both genotypes.

\section{ECS parameters in vitro}

The experimental arrangement for in vitro diffusion measurements is shown in Figure $2 \mathrm{~A}$. Recordings were taken in the same sequence as described for the in vivo preparation.

Representative diffusion curves superimposed with fitted theoretical curves for that record are shown in Figure $2 \mathrm{~B}$. Again, the amplitude of the theoretical diffusion curve (Fig. 2C) based on the mean data (Table 1 ) was smaller in AQP4 ${ }^{-1-}$ than in AQP4 ${ }^{+/+}$mice in vitro, whereas the scaled theoretical curves did not differ (Fig. 2C, inset).

Six brain slices from three AQP4 ${ }^{+/+}$mice and six brain slices 
Table 1. Extracellular volume fraction, tortuosity, and $\mathrm{TMA}^{+}$loss in the somatosensory neocortex of AQP4 ${ }^{+/+}$ and $A Q P 4^{-1-}$ mice

\begin{tabular}{llllll}
\hline Genotype & Preparation & $\alpha$ & $\lambda$ & $k^{\prime}\left(s^{-1}\right)$ & $n$ \\
\hline $\mathrm{AQP4}^{+/+}$ & In vivo & $0.18 \pm 0.003$ & $1.61 \pm 0.02$ & $0.0031 \pm 0.0009$ & 7 \\
$\mathrm{AQP4}^{-1-}$ & & $0.23 \pm 0.007^{*}$ & $1.62 \pm 0.04$ & $0.0045 \pm 0.0001$ & 7 \\
$\mathrm{AQP4} 4^{+1+}$ & In vitro & $0.19 \pm 0.002$ & $1.61 \pm 0.03$ & $0.013 \pm 0.001$ & 6 \\
$\mathrm{AQP4}^{-1-}$ & & $0.23 \pm 0.003^{*}$ & $1.64 \pm 0.01$ & $0.013 \pm 0.001$ & 6 \\
\hline
\end{tabular}

Data are expressed as mean \pm SEM. $n$ is the number of animals (in vivo) or brain slices (in vitro). *Statistically significant difference $(p<0.001$, two-sample equal-variance $t$ test) between $A Q P 4^{+/+}$and $A Q P 4^{-/-}$.

from three AQP4 ${ }^{-1-}$ mice were used. In each slice, 2-5 diffusion curves were obtained, and the parameters were averaged to yield one set of $\alpha, \lambda$, and $k^{\prime}$ per slice with summary values shown in Figure $2 D$ and Table 1 . The in vitro results closely resembled those in vivo.

\section{Discussion}

Using the well established RTI-TMA method in the somatosensory cortex of AQP4-deficient mice under resting physiological conditions, we found a significant increase of $28 \%$ in ECS volume fraction, $\alpha$, compared with wild type in vivo, and this was confirmed in brain slices (increase of $21 \%$ ). There was no difference, however, in ECS tortuosity, $\lambda$, between AQP4 ${ }^{-1-}$ mice and their WT counterparts. Loss of TMA ${ }^{+}$, characterized by $k^{\prime}$, was also unchanged between the two genotypes. Our data enable us to evaluate some of the speculations arising from other recent studies of AQP4 ${ }^{-1-}$ mice.

\section{Comparison with other wild-type mouse studies}

The RTI-TMA method is the only technique available today for simultaneous measurement of the absolute values of $\alpha, \lambda$, and $k^{\prime}$ in vivo as well as in vitro (Nicholson and Phillips, 1981; Nicholson, 1993). Values of $\alpha(0.18-0.19)$ and $\lambda(1.61)$ found in wildtype mice in the present study can be compared with other in vivo studies on wild-type mouse cortex. For ages 3-6 months, Anděrová et al. (2001) found $\alpha=0.23, \lambda=1.67$; for ages $6-8$ months, Syková et al. (2005a) reported $\alpha=0.20, \lambda=1.47-1.50$; for ages 5-9 months, Syková et al. (2005b) reported $\alpha=0.17$, $\lambda=1.57$. Variations among mouse strains probably account for the small differences in values. Loss of TMA ${ }^{+}$from the ECS in vivo in our study was comparable with typical values of $k^{\prime}=$ $3-10 \times 10^{-3} \mathrm{~s}^{-1}$ in the three studies referenced above, and the increased value measured here in vitro is likely caused by escape of $\mathrm{TMA}^{+}$at the slice surface (Hrabětová and Nicholson, 2007).

\section{Implications of the increased volume fraction in $\mathrm{AQP}^{-1-}$ mice}

It has been assumed that the development of the mouse cortex resembles that of the rat (Lehmenkühler et al., 1993) and therefore that $\alpha$ is expanded to $\sim 0.4$ in the neonate (Wen et al., 1999). Therefore, our results imply that the ECS is reduced to approximately one-half of this value as the animal matures in both $\mathrm{AQP} 4^{+/+}$and AQP4 ${ }^{-/-}$mice, which indicates that AQP4 channels are not obligatory for this contraction.

The increased $\alpha$ in the AQP4-deficient mice compared with wild type cannot be attributed to a reduction in the volume of the astrocytic endfeet, where the AQP4 channels are most concentrated, because there is no change in endfoot cross-section area in AQP4 ${ }^{-1-}$ mice compared with AQP4 ${ }^{+/+}$(Manley et al., 2000). Other studies have demonstrated that AQP4 facilitates the removal of excess brain water in models of vasogenic edema in which water accumulates in the ECS (Papadopoulos et al., 2004).
Thus, it is possible that lack of AQP4 might alter ECS water homeostasis, resulting in the expansion of baseline $\alpha$.

Given that $\alpha$ in $\mathrm{AQP} 4^{-/-}$mice was $\sim 28 \%$ greater than in $\mathrm{AQP} 4{ }^{+/+}$mice, we may ask whether this might have functional consequences, for example, in the known increase in seizure threshold (Binder et al., 2004a, 2006). Several in vitro studies have shown that application of hyperosmolar solutions containing impermeable substances elevates the seizure threshold in rat hippocampus (Traynelis and Dingledine, 1989; Dudek et al., 1990; Pan and Stringer, 1996; Kilb et al., 2006). Hyperosmolar solutions should increase $\alpha$ by shrinking cells, and Traynelis and Dingledine (1989) confirmed this through impedance measurements. These impedance data [Traynelis and Dingledine (1989), their Table 2] correspond to a 1-10\% increase in $\alpha$ (assuming $\alpha$ is proportional to impedance). This estimate can be corroborated using the osmotic data of Traynelis and Dingledine (1989) and Equation 13 of Kume-Kick et al. (2002) that predicts a $2-13 \%$ increase in $\alpha$. Thus, even a moderate expansion of the ECS may be sufficient to increase the threshold for seizure by reducing the buildup of neuroactive substances in the ECS or decreasing ephaptic interactions that normally promote synchronized firing. Prolonged duration of seizure is more likely related to $\mathrm{K}^{+}$clearance, because this is impaired in both AQP4 ${ }^{-1-}$ (Binder et al., 2006) and $\alpha$-Syn ${ }^{-1-}$ (AmiryMoghaddam et al., 2003) mice. Such clearance may require water transport or functional coupling of AQP4 and potassium channels (Nagelhus et al., 2004).

\section{Tortuosity is unchanged in AQP4 $4^{-/-}$mice}

Our finding that tortuosity is unaltered in AQP4 ${ }^{-1-}$ mice compared with the wild type is in contrast to data obtained with fluorescence recovery after photobleaching (FRAP). Using fluorescent dextrans (predominantly $70 \mathrm{kDa}$ ) and two FRAP methods, $\lambda$ was found to be $10-20 \%$ less in AQP4 ${ }^{-1-}$ mouse neocortex than in wild type in undisturbed conditions in vivo (Binder et al., 2004b; Papadopoulos and Verkman, 2005; Zador et al., 2008). The $70 \mathrm{kDa}$ dextran molecule is considerably larger than $\mathrm{TMA}^{+}$, and this might be a factor, but further studies with macromolecules will be required to resolve this discrepancy with our results.

Whether or not it is verified that $\lambda$ is reduced in AQP4 ${ }^{-1-}$ mice compared with wild type when dextrans are used, the assumption that this implies an increase in $\alpha$ (Binder et al., 2004b; Papadopoulos and Verkman, 2005; Zador et al., 2008) is not justified. With the definition of tortuosity used in the RTI-TMA and FRAP techniques, $\lambda$ and $\alpha$ are independent parameters (Nicholson and Phillips, 1981; Lehmenkühler et al., 1993; KumeKick et al., 2002), and this is borne out by the present data. A dependency may only be derived for some highly constrained models of the ECS (Tao and Nicholson, 2004; Hrabe et al., 2004), and such models do not exist for the complex cytoarchitectonics of the cerebral cortex.

In conclusion, our results show that there is an expansion of the ECS in AQP4 ${ }^{-1-}$ mice compared with wild type; although this probably is inadequate to explain all the differences in pathophysiology seen between these two AQP4 genotypes, the expansion may account for the increased threshold for seizure in AQP4-deficient mice. Furthermore, the data we present here will provide useful parameters for modeling ion and water transport in brain tissue. 


\section{References}

Amiry-Moghaddam M, Williamson A, Palomba M, Eid T, de Lanerolle NC, Nagelhus EA, Adams ME, Froehner SC, Agre P, Ottersen OP (2003) Delayed $\mathrm{K}^{+}$clearance associated with aquaporin-4 mislocalization: phenotypic defects in brains of alpha-syntrophin-null mice. Proc Natl Acad Sci USA 100:13615-13620.

Anděrová M, Kubinová S, Mazel T, Chvátal A, Eliasson C, Pekny M, Syková E (2001) Effect of elevated $\mathrm{K}^{+}$, hypotonic stress, and cortical spreading depression on astrocyte swelling in GFAP-deficient mice. Glia 35:189-203.

Binder DK, Oshio K, Ma T, Verkman AS, Manley GT (2004a) Increased seizure threshold in mice lacking aquaporin-4 water channels. NeuroReport 15:259-262.

Binder DK, Papadopoulos MC, Haggie PM, Verkman AS (2004b) In vivo measurement of brain extracellular space diffusion by cortical surface photobleaching. J Neurosci 24:8049-8056.

Binder DK, Yao X, Zador Z, Sick TJ, Verkman AS, Manley GT (2006) Increased seizure duration and slowed potassium kinetics in mice lacking aquaporin-4 water channels. Glia 53:631-636.

Dudek FE, Obenaus A, Tasker JG (1990) Osmolality-induced changes in extracellular volume alter epileptiform bursts independent of chemical synapses in the rat: importance of non-synaptic mechanisms in hippocampal epileptogenesis. Neurosci Lett 120:267-270.

Franklin KBJ, Paxinos G (2008) The mouse brain in stereotaxic coordinates, Ed 3. San Diego: Elsevier.

Frigeri A, Gropper MA, Umenishi F, Kawashima M, Brown D, Verkman AS (1995) Localization of MIWC and GLIP water channel homologs in neuromuscular, epithelial and glandular tissues. J Cell Sci 108:2993-3002.

Hrabe J, Hrabětová S, Segeth K (2004) A model of effective diffusion and tortuosity in the extracellular space of the brain. Biophys J 87:1606-1617.

Hrabětová S, Nicholson C (2007) Biophysical properties of brain extracellular space explored with ion-selective microelectrodes, integrative optical imaging and related techniques. In: Electrochemical methods for neuroscience (Michael AC, Borland LM, eds), pp 167-204. Boca Raton, FL: CRC, Taylor Francis Group.

Hrabětová S, Hrabe J, Nicholson C (2003) Dead-space microdomains hinder extracellular diffusion in rat neocortex during ischemia. J Neurosci 23:8351-8359.

Kilb W, Dierkes PW, Syková E, Vargová L, Luhmann HJ (2006) Hypoosmolar conditions reduce extracellular volume fraction and enhance epileptiform activity in the CA3 region of the immature rat hippocampus. J Neurosci Res 84:119-129.

Kume-Kick J, Mazel T, Voříšek I, Hrabětová S, Tao L, Nicholson C (2002) Independence of extracellular tortuosity and volume fraction during osmotic challenge in rat neocortex. J Physiol (Lond) 542:515-527.

Lehmenkühler A, Syková E, Svoboda J, Zilles K, Nicholson C (1993) Extracellular space parameters in the rat neocortex and subcortical white matter during postnatal development determined by diffusion analysis. Neuroscience 55:339-351.

Ma T, Yang B, Gillespie A, Carlson EJ, Epstein CJ, Verkman AS (1997) Gen- eration and phenotype of a transgenic knockout mouse lacking the mercurial-insensitive water channel aquaporin-4. J Clin Invest 100:957-962.

Manley GT, Fujimura M, Ma T, Noshita N, Filiz F, Bollen AW, Chan P, Verkman AS (2000) Aquaporin-4 deletion in mice reduces brain edema after acute water intoxication and ischemic stroke. Nat Med 6:159-163.

Manley GT, Binder DK, Papadopoulos MC, Verkman AS (2004) New insights into water transport and edema in the central nervous system from phenotype analysis of aquaporin-4 null mice. Neuroscience 129:983-991.

Nagelhus EA, Mathiisen TM, Ottersen OP (2004) Aquaporin-4 in the central nervous system: cellular and subcellular distribution and coexpression with KIR4.1. Neuroscience 129:905-913.

Nicholson C (1993) Ion-selective microelectrodes and diffusion measurements as tools to explore the brain cell microenvironment. J Neurosci Methods 48:199-213.

Nicholson C, Phillips JM (1981) Ion diffusion modified by tortuosity and volume fraction in the extracellular microenvironment of the rat cerebellum. J Physiol (Lond) 321:225-257.

Nielsen S, Nagelhus EA, Amiry-Moghaddam M, Bourque C, Agre P, Ottersen OP (1997) Specialized membrane domains for water transport in glial cells: high-resolution immunogold cytochemistry of aquaporin-4 in rat brain. J Neurosci 17:171-180.

Pan E, Stringer JL (1996) Influence of osmolality on seizure amplitude and propagation in the rat dentate gyrus. Neurosci Lett 207:9-12.

Papadopoulos MC, Verkman AS (2005) Aquaporin-4 gene disruption in mice reduces brain swelling and mortality in pneumococcal meningitis. J Biol Chem 280:13906-13912.

Papadopoulos MC, Manley GT, Krishna S, Verkman AS (2004) Aquaporin 4 facilitates the reabsorption of excess fluid in vasogenic brain edema. FASEB J 18:1291-1293.

Syková E, Voříšek I, Antonova T, Mazel T, Meyer-Luehmann M, Jucker M, Hájek M, Or M, Bureš J (2005a) Changes in extracellular space size and geometry in APP23 transgenic mice: a model of Alzheimer's disease. Proc Natl Acad Sci USA 102:479-484.

Syková E, Voř́šek I, Mazel T, Antonova T, Schachner M (2005b) Reduced extracellular space in the brain of tenascin-R- and HNK-1sulphotransferase deficient mice. Eur J Neurosci 22:1873-1880.

Tao L, Nicholson C (2004) Maximum geometrical hindrance to diffusion in brain extracellular space surrounding uniformly spaced convex cells. J Theoret Biol 229:59-68.

Traynelis SF, Dingledine R (1989) Role of extracellular space in hyperosmotic suppression of potassium-induced electrographic seizures. J Neurophysiol 61:927-938.

Wen H, Nagelhus EA, Amiry-Moghaddam M, Agre P, Ottersen OP, Nielsen S (1999) Ontogeny of water transport in rat brain: postnatal expression of the aquaporin-4 water channel. Eur J Neurosci 11:935-945.

Zador Z, Magzoub M, Jin S, Manley GT, Papadopoulos MC, Verkman AS (2008) Microfiberoptic fluorescence photobleaching reveals sizedependent macromolecule diffusion in extracellular space deep in brain. FASEB J 22:870-879. 